\title{
Aesthetic and Non-aesthetic Indications for Orbicularis Oculi Myectomy
}

\author{
Fausto Viterbo $^{1} \cdot$ Janna Joethy $^{2} \cdot$ Ryane Schmidt Brock $^{3}$
}

Received: 11 October 2015/Accepted: 16 March 2016/Published online: 13 May 2016

(C) Springer Science+Business Media New York and International Society of Aesthetic Plastic Surgery 2016

\begin{abstract}
Introduction Crows' feet wrinkles are caused by the action of the orbicularis oculi muscle on overlying skin. Treatment options range from botulinum toxin to a multitude of surgical methods. We first described our technique in 2003 and refined it in 2006. We highlight the evolution of our technique and look at our results to assess the effectiveness of our technique.

Objective The aim of this article is to show our experience of 13 years in 134 patients with our technique of orbicularis oculi myectomy.

Methods From September 2000 to July 2013, we operated on 134 patients with an age range of 28-77 years. Of these patients, 104 had myectomies via lifting and 22 via a blepharoplasty approach. Five patients had myectomies for treatment of blepharospasm and a further three patients to restore symmetry in facial palsy. An evaluation of the results was performed by two medical students.

Results Our results showed reduction of the wrinkles in all cases. For the statistical analysis the Wilcoxon test was performed. The $p$ value was less than 0.001 showing a
\end{abstract}

Electronic supplementary material The online version of this article (doi:10.1007/s00266-016-0638-5) contains supplementary material, which is available to authorized users.

Fausto Viterbo

fv@faustoviterbo.com.br

1 Department of Plastic Surgery, São Paulo State University Julio de Mesquita Filho, UNESP, Rua Domingos Minicucci Filho, 587, Botucatu, São Paulo 18607-030, Brazil

2 Department of Plastic, Reconstructive and Aesthetic Surgery, Singapore General Hospital, Outram Road, Singapore, Singapore

3 Brazilian Plastic Surgery Society, São Paulo, Brazil significant reduction of crows' feet wrinkles in both sides when the orbicularis myectomy was performed, via blepharoplasty or lifting. For myectomy performed for blepharospasm or facial palsy, the statistical analysis was not done due to the low numbers.

Conclusion In the appropriate patient, orbicularis oculi myectomy is an effective and long-term treatment for crows' feet wrinkles with a low risk of complications and high patient satisfaction.

Level of Evidence IV This journal requires that authors assign a level of evidence to each article. For a full description of these Evidence-Based Medicine ratings, please refer to the Table of Contents or the online Instructions to Authors www.springer.com/00266.

Keywords Crows' feet wrinkles · Orbicularis oculi · Myectomy $\cdot$ Botulinum toxin

\section{Introduction}

The search for a youthful appearance is deeply ingrained in modern society. One of the first signs of ageing is crows' feet wrinkles. These wrinkles are caused by the action of the orbicularis oculi muscle on the overlying skin [1].

There are several kinds of treatments based on the type of the wrinkles. Park et al. [2] used the definition proposed by Lemperle et al. [3] to evaluate their results using photo shoots. Four types of wrinkles have been classified by cause and depth of invasion and have been studied using two-dimensional photography [4]. Cula et al. [5] developed an algorithm for automatic detection of facial wrinkles based on estimating the orientation and the frequency of elongated spatial features, captured by digital image filtering. Fujimura et al. [6] studied the mechanism of wrinkle 
formation by investigating the relationship between the movement of skin and wrinkle formation using particle image velocimetry analysis.

The simplest treatment for crows' feet wrinkles is botulinum toxin injection. This technique brings effective but ephemeral results as the botulinum toxin loses its efficacy and thus needs to be repeated periodically [7].

Of the surgical options, Skoog noted that the resection of a small portion of the orbicularis oculi could result in improvement of wrinkles, but this was not described [8]. Via a facelift approach, Aston detached the lateral portion of the muscle and used stitches to keep the muscle splayed out [9]. Connell modified Aston's technique by making a small incision in the lateral border of this muscle allowing the orbicularis to be splayed a little more [9, 10]. Camirand described resection of a horizontal rectangle of orbicularis muscle via a blepharoplasty approach [11]. Also via a blepharoplasty approach, Bonatto et al. resected more muscle by performing a trapezoidal myectomy [12]. Other authors have described using orbicularis muscle flaps to provide superior traction to improve the periorbital region [1315]. Another description to improve crows' feet is performed by detaching and suspending the orbicularis oculi muscle with partial denervation [16]. Although all these contributions are important, we feel that they do not provide long-term or satisfactory reduction of Crow's feet.

In 2003, Viterbo introduced the vertical resection of the lateral portion of the orbicularis oculi muscle, removing just enough muscle to eliminate the wrinkles when the patient smiles [17]. The amount removed was an average length of $3.25 \mathrm{~cm}$. This procedure was done via a facelift or blepharoplasty approach and produced effective and long-lasting results. An improvement of this technique was published in 2006 when Viterbo and Lutz described more extensive resection of the orbicularis, performing the myectomy in a " $\mathrm{C}$ " shape in the direction of the muscle fibres, which resulted in a more effective and definitive result [18]. Carvalho et al. also performed this technique and reported satisfactory results [19].

Another way to treat crows' feet wrinkles is through radiofrequency applied directly to the exposed muscle during a facelift to promote irreversible fibrosis of this muscle [20]. The use of radiofrequency for muscle reduction is not new and has been described before [21].

We believe that the orbicularis oculi myectomy is the most effective and long-lasting method and therefore important in achieving facial rejuvenation.

The aim of this article is to show our experience with the orbicularis oculi myectomy technique in 134 patients over a period of 13 years.

\section{Method}

From September 2000 to July 2013, we operated on 134 patients, 111 women and 23 men. Ages ranged between 28 and 77 years with an average age of 53 years $( \pm 9.4)$. Of these patients, 104 had myectomies via lifting (89 via cervico-facial-temporal lifting and 15 via temporal lifting) and 22 through a blepharoplasty approach. Five patients had myectomies for treatment of blepharospasm and a further 3 patients for symmetry in facial palsy management.

Prior to surgery, crow's feet are marked by getting patients to forcefully smile and rest (Fig. 1). For cervicofacial-temporal lifting, we start with a subcutaneous detachment (Fig. 2). At the temporal portion, Loupe magnification $(3 \times)$ is used to undermine the skin till the lateral edge of the orbicularis muscle is reached. The area previously marked in the skin (Fig. 2) is now transposed to the muscle (Fig. 3). With a pair of fine scissors, muscle resection is performed (Fig. 4). Muscle can be removed "en bloc" or "in strips" (Supplementary Movies 1 and 2). Extra attention is required to dissect just under the muscle, preserving underlying nerves. If these nerves are damaged, paralysis of the upper eyelid can be a consequence. Any muscle that is located over or medial to the orbital rim is preserved to maintain eyelid sphincter mechanisms.

After muscle removal, haemostasis is achieved using low-voltage bipolar electrocautery, again avoiding nerve injury in this region. Skin is then allowed to re-drape and we evaluate the need for volume replacement (Supplementary Movie 3).

Previously, when volume replacement was required, we harvested a rectangular-shaped piece of SMAS from the pre- and infra-auricular region and placed it in the area where the myectomy was performed. Now, we inject fat where the myectomy is performed (Fig. 5). In most cases,
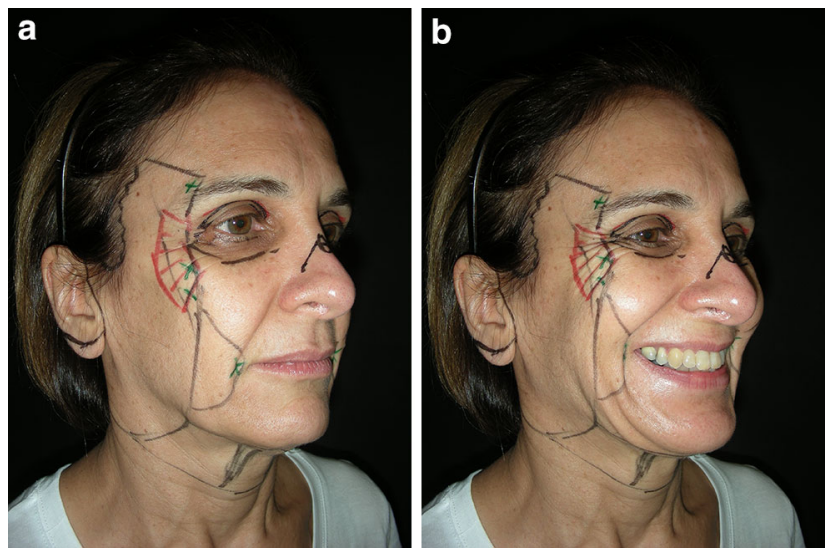

Fig. 1 a Preoperative patient in repose. b Smiling preoperative patient showing crows' feet wrinkles 


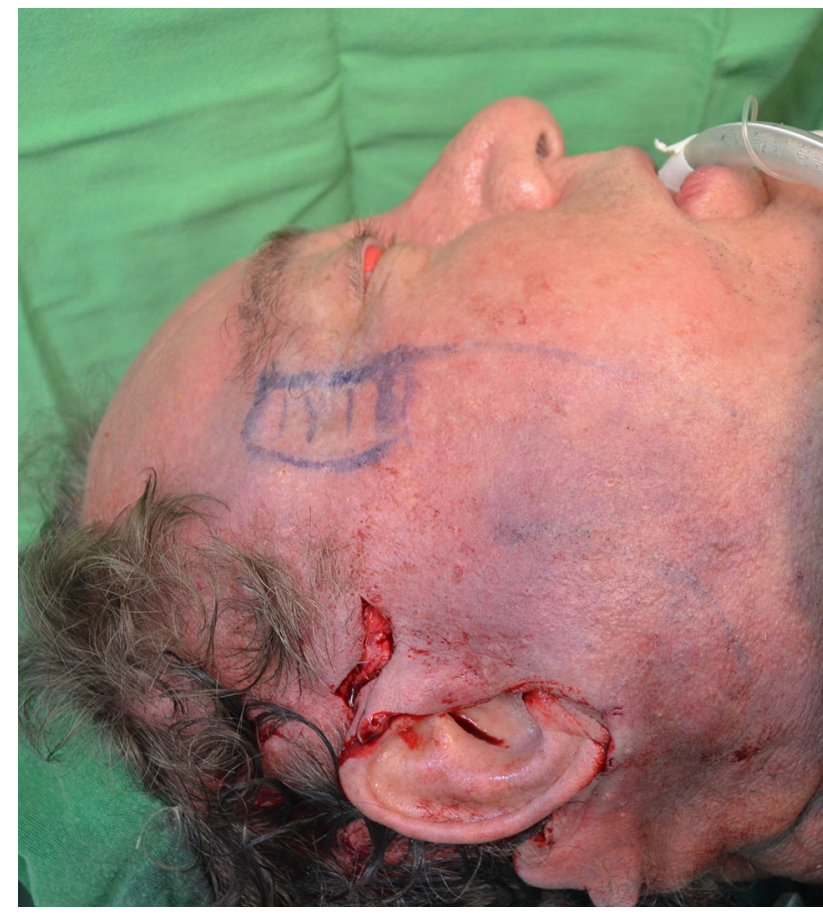

Fig. 2 The portion of the orbicularis oculi muscle to be removed was marked on the skin

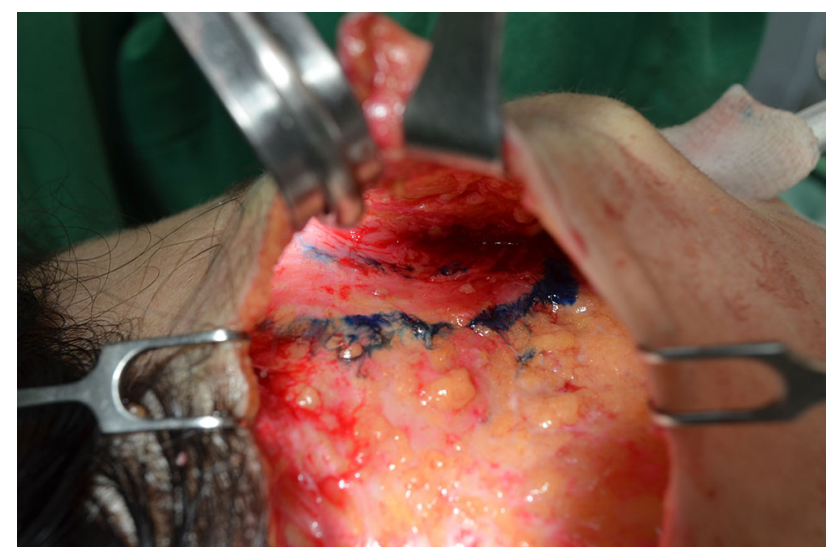

Fig. 3 The portion of the orbicularis oculi muscle to be removed was marked with methylene blue taking the cutaneous markings into account

we inject $3-5 \mathrm{ml}$ of aspirated fat in the tissue beneath the excised muscle (Fig. 6).

When cervico-facial lifting is performed, visualization of the superior portion of the orbicularis oculi area becomes more difficult. In these cases, we undermine the hair-bearing temporal region and approach the muscle from the superior portion. This procedure leaves minimal scaring and does not displace the hair superiorly (Fig. 7).

In temporal lifting, the cutaneous detachment is performed in the cranial-caudal direction, and the

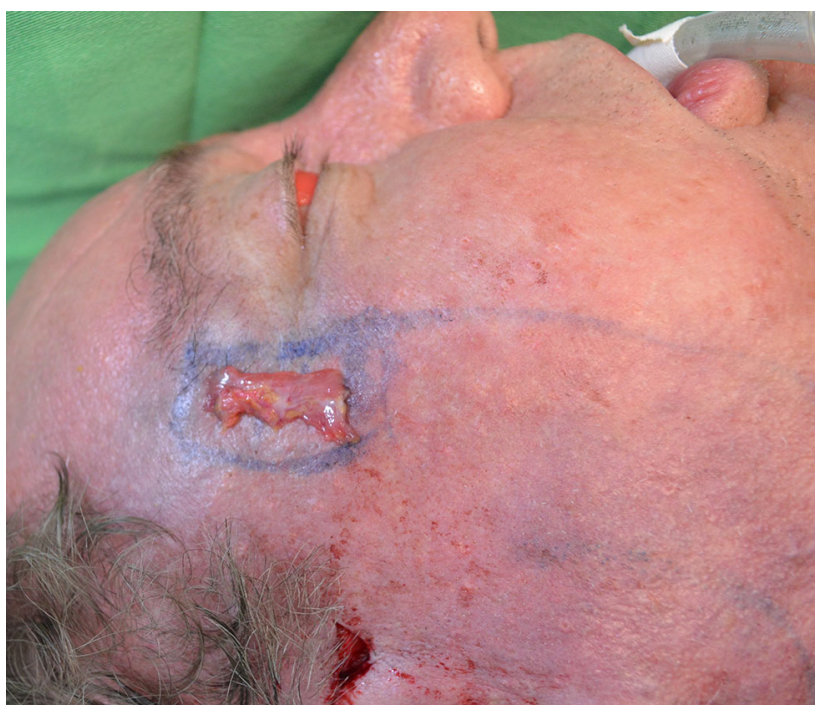

Fig. 4 Excised portion of the orbicularis oculi muscle placed on top of the skin

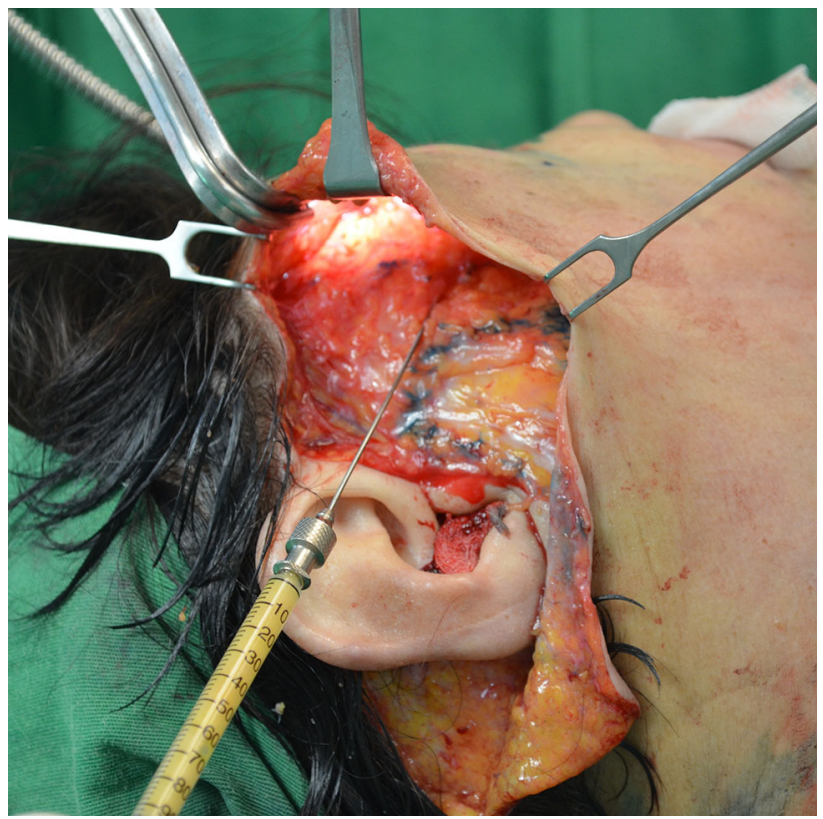

Fig. 5 Aspirated fat is injected in the tissue under the myectomy to prevent depression

visualization of the inferior portion of the muscle becomes a little more difficult. This technique requires more attention and a long-lighted retractor. After muscle removal, if there is depression, fat is injected. When aspirated fat is used, we place micropore tapes on the skin in a vertical and oblique way to keep the fat in position and evenly distributed.

Myectomy via blepharoplasty can be performed via upper or lower eyelid incisions but is easier to be performed with both approaches. This enables the superior and 
Fig. 6 a Schematic showing the muscle in proximity to the frontal branch of the facial nerve, the zygomaticotemporal and the zygomaticofacial nerves. The dotted area represents the area of muscle that is excised. b Schematic after muscle excision. This area is typically injected with fat. Note that muscle fibres medial to the orbital rim are not excised
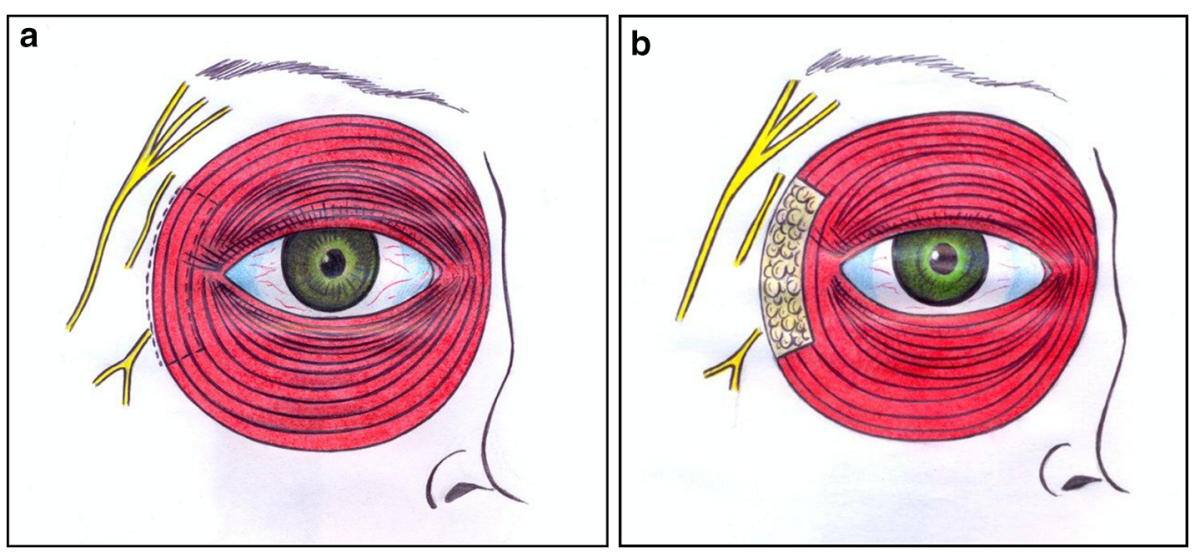

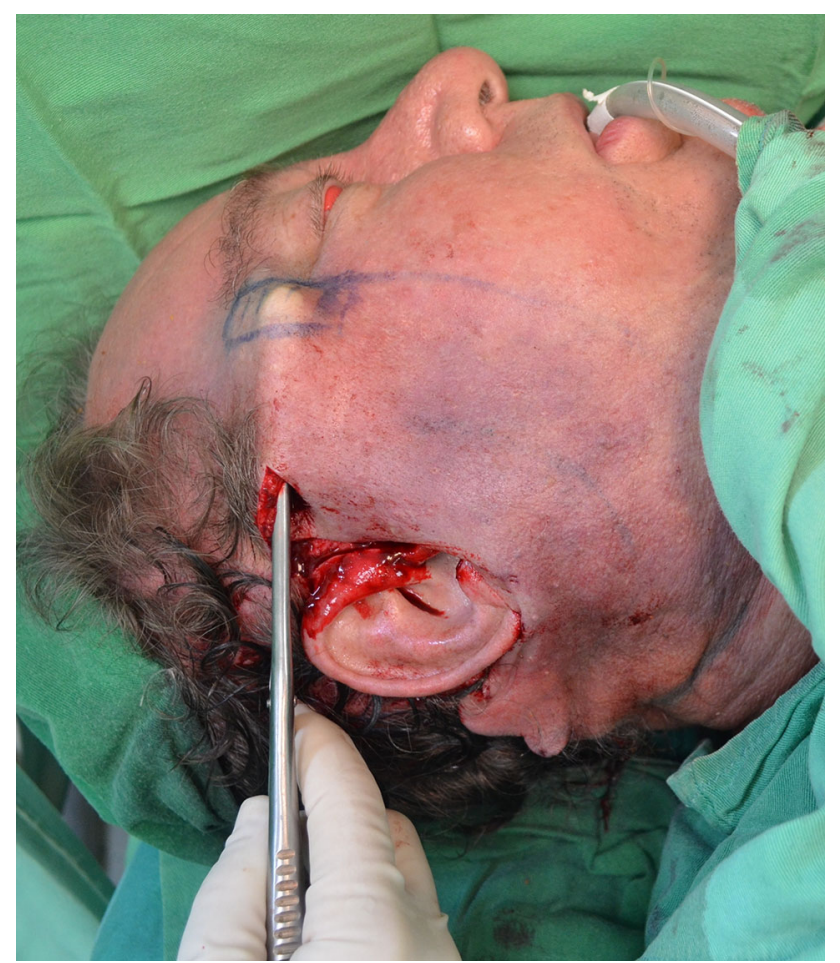

Fig. 7 Subcutaneous elevation in the temporal region is done with Viterbo dissectors to approach the muscle from the superior aspect, without enlarging the incision. All the skin above the orbicularis oculi muscle is detached

inferior portion of the muscle to be well visualized. Dissection starts superiorly through the upper eyelid with skin elevation leaving the muscle to be resected exposed. After careful elevation of the muscle, it is sharply divided preserving the medical muscle fibres that are located over the orbital rim to maintain sphincteric function. If volume is required, fat is injected.

In the first three postoperative days, we recommend cold and wet compression to the eyes. For our lifting procedures, we routinely use a drain for $24-48 \mathrm{~h}$ after the surgery. The eyelid sutures are usually removed after 5 days, and the lifting sutures after 10 days. We prescribe Cefuroxime $250 \mathrm{mg}$, twice a day for 5 days.

An evaluation of the results was performed by two medical students who had no knowledge about the technique used. They evaluated pre- and 6 month postoperative photographs of the patients smiling and analysed the quantity of wrinkles in the lateral region of the orbit, rating as zero when no wrinkles were visible and 10 when many wrinkles were visible.

\section{Results}

The average follow-up period was 5.3 years ( 3 months to 11.1 years). All patients had reductions in crows' feet with no impairment of lid movement (Supplementary Movies 4-7).

The evaluation of the quantity of wrinkles in the lateral region of the orbit in the smiling pictures of the patients submitted to myectomy via cervico-facial lifting showed an average score of $4.82( \pm 0.44)$ in the preoperative photographs and $1.60( \pm 0.66)$ in the postoperative photos, with an improvement of $66.8 \%$. Using the temporal approach, the average score was $4.17( \pm 0.59)$ in the preoperative compared to $0.71( \pm 0.38)$ in the postoperative photographs, with an improvement of $82.9 \%$. For blepharoplasty, the average was $4.49( \pm 0.29)$ in the preoperative versus 1.85 $( \pm 0.28)$ in the postoperative photographs, with an improvement of $58.7 \%$. In myectomy for blepharospasm, the average was $3.83( \pm 0.28)$ in the preoperative and 1 $( \pm 0)$ in the postoperative photographs, with an improvement of $73.8 \%$. When the myectomy was done in the healthy side for facial palsy symmetry, the average score was $3.87( \pm 1.1)$ in preoperative photographs and 1.04 $( \pm 0.88)$ in postoperative photographs, with an improvement of $73.1 \%$ (Table 1).

The results showed reduction of wrinkles in all the cases. In some cases, these wrinkles disappeared almost 
Table 1 Average of the crows' feet wrinkle evaluation followed by the standard deviation, the maximum and minimum scores, and the percentage of improvement

\begin{tabular}{llll}
\hline & Pre-op & Post-op & Improvement $(\%)$ \\
\hline Cervico-facial lifting & $4.82( \pm 0.44)$ & $1.60( \pm 0.66)$ & 66.8 \\
Temporal lifting & $(10-1)$ & $(9-0)$ & $0.71( \pm 0.38)$ \\
& $4.17( \pm 0.59)$ & $(9-0)$ & 82.9 \\
Blepharoplasty & $(9-1)$ & $1.85( \pm 0.28)$ & 58.7 \\
Blepharospasm treatment & $4.49( \pm 0.29)$ & $(7-0)$ & $7( \pm 0)$ \\
Symmetry for facial palsy & $(9-2)$ & $(1-1)$ & 73.8 \\
& $3.83( \pm 0.28)$ & $1.04( \pm 0.88)$ & 73.1 \\
\hline
\end{tabular}

completely (Figs. 8, 9), whereas in other cases some of them remained, especially in the lower part (Figs. 10, 11 and Supplementary Movies 4-7). For the statistical analysis, the Wilcoxon test was performed. The " $p$ " value was less than 0.001 showing a significant reduction of the crows' feet wrinkles in both sides when the orbicularis myectomy was performed, via blepharoplasty or lifting. For the blepharospasm and facial palsy procedures, the statistical analysis could not be performed due to the low number of patients.

In terms of limitations and complications, 4 patients in the lifting group had postoperative haematoma that resolved spontaneously. Of the 134 patients, 11 had remnant lines in the lower area of the crows' feet. Remnant lower rhytids were more common when the blepharoplasty approach was used (Fig. 11). Typically, excess lower
Fig. 8 Preoperative views of a 51-year-old patient at repose (a), smiling (b), and $45^{\circ}$ view (c). One year and 4 months post orbicularis myectomy via cervico-facial-temporal lifting at repose (d), smiling (e), and $45^{\circ}$ view (f)
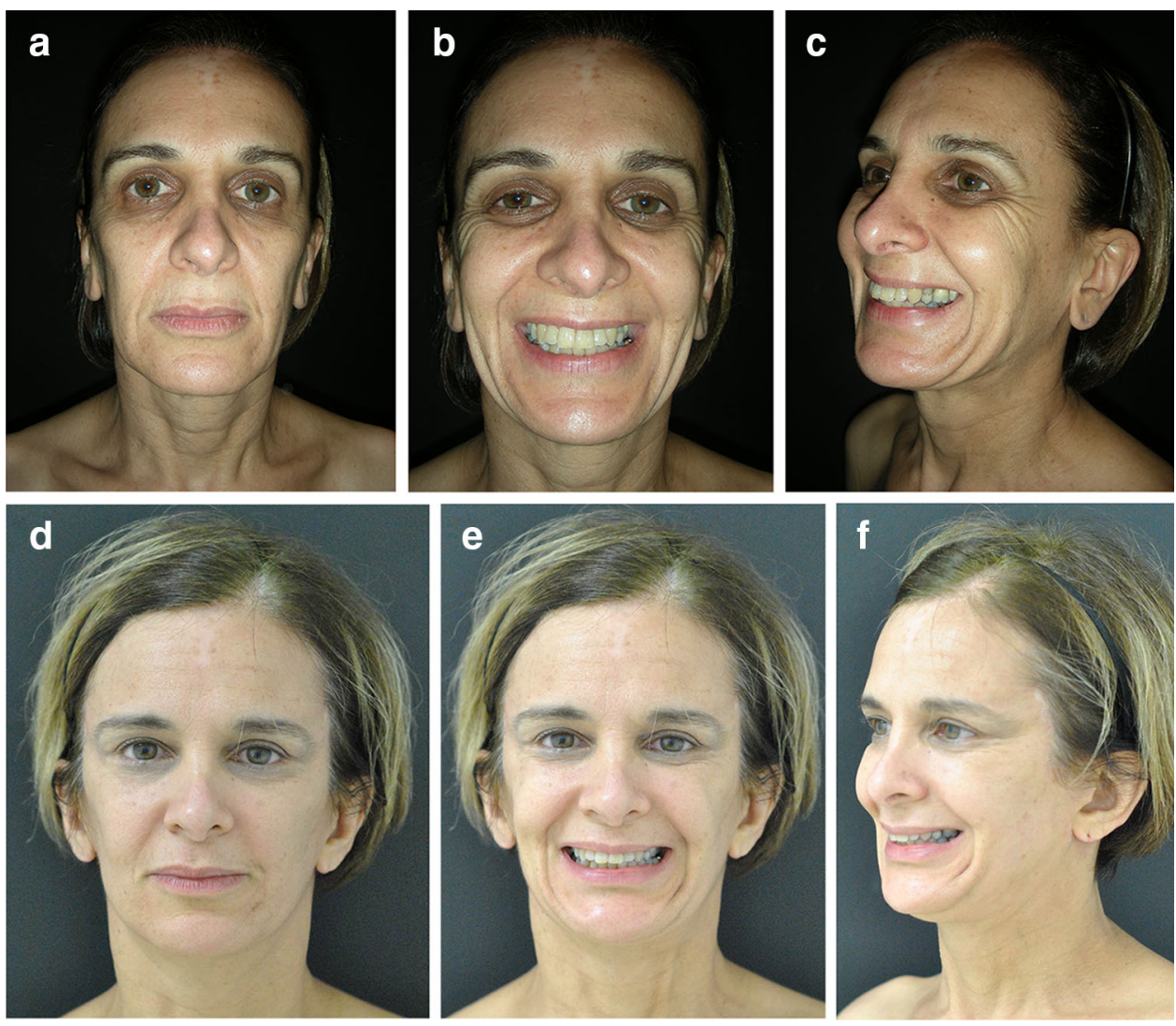
Fig. 9 Preoperative views of a 36-year-old patient at repose (a), smiling (b), and $45^{\circ}$ view (c). Eight years and 4 months post orbicularis myectomy via cervico-facial-temporal lifting at repose (d), smiling (e), and $45^{\circ}$ view (f)
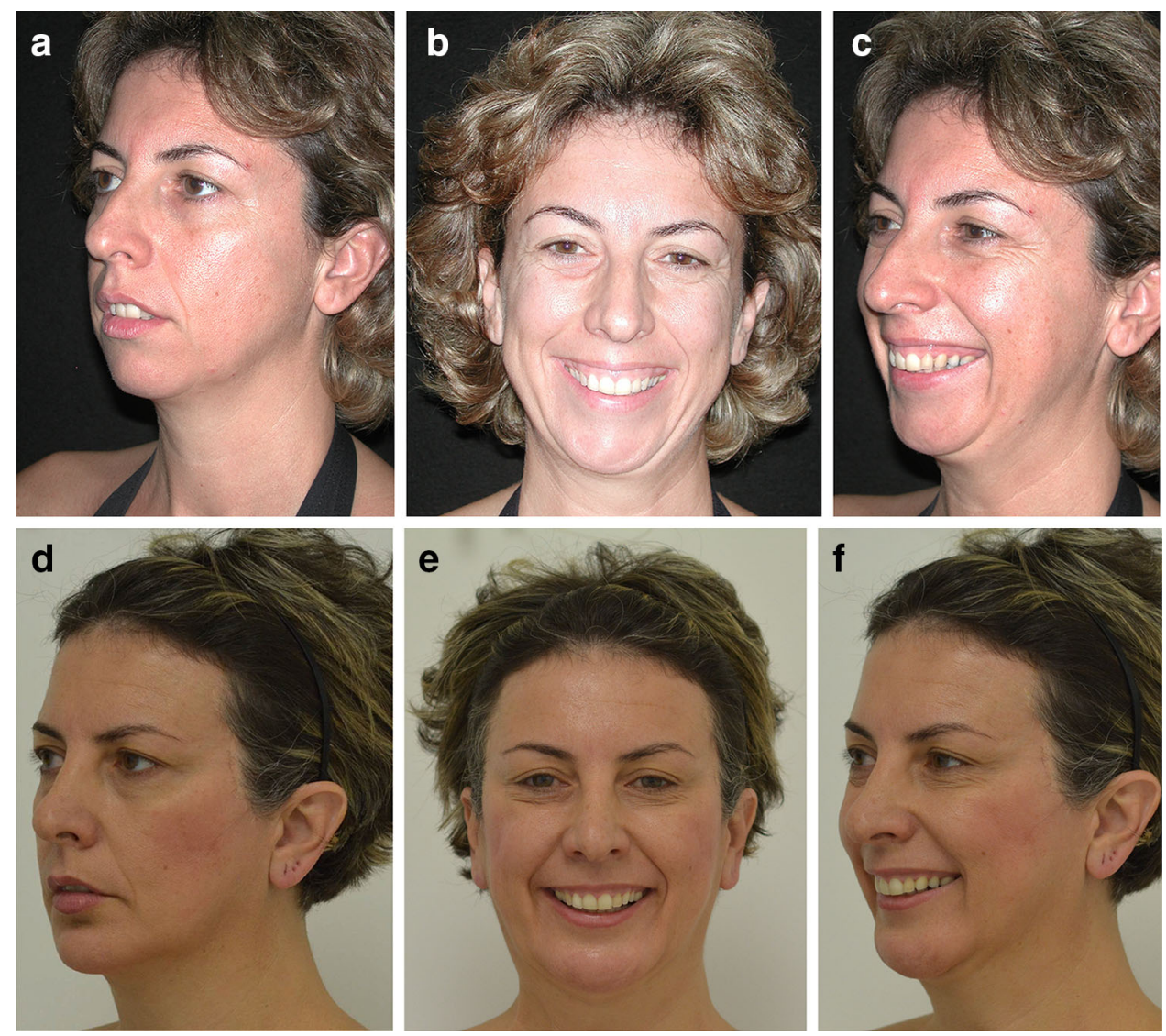

eyelid skin is a transient problem in our patients. This could be due perhaps to the diminished muscle insertion into the dermis (resulting from our muscle excision and mobilization of overlying skin) or inadequate skin resection during blepharoplasty. Furthermore, we feel that perhaps elimination of rhytids on the lateral aspect (crows' feet) tends to draw attention to the lower eyelid rhytids. However, we found that in myectomies performed via blepharoplasty and in those patients who have significant wrinkles extending to the inferior portion, less improvement was noted at the lower region. No lagophthalmos was noted and we feel that this is reflective of our surgical technique.

Three patients had too much fat placed and underwent liposuction for removal of fat. Three patients had obvious hollowing and two of these patients underwent delayed fat grafting. One patient with hollowing is shown in Fig. 12. One patient had puckering (Fig. 13) noticed more when smiling but we managed this conservatively by asking the patient to massage with improvement seen after 9 months.

Chemosis was a frequent complication and was more notable in the group that had myomectomy preformed via a blepharoplasty approach. Patients are warned that chemosis can last for a month. Bruising was seen in almost all our cases and patients were warned preoperatively to anticipate this. Six patients were initially concerned about their scar but all 6 patients were satisfied by 6 months.

\section{Discussion}

The great majority of our patients that seek rejuvenation present with crows' feet wrinkles. We offer two options, botulinum toxin and orbicularis myectomy, explaining the advantages and disadvantages of each one. Some patients prefer a less invasive treatment and choose botulinum toxin, but some chose the surgical procedure, due to the temporary results and high cost of repeated botulinum toxin injections.

We do not have experience with muscle cauterization, but we caution that monopolar cautery may result in nerve injury. We also do not have experience with radiofrequency, but this procedure requires special equipment that may add to cost. For these methods to be effective, they should cause a fibrosis in the muscle that may lead to muscle atrophy. This muscle atrophy can lead to depression and we suspect that some cases will require fat grafting. 
Fig. 10 Preoperative views of a 54-year-old patient at repose (a), smiling (b), and $45^{\circ}$ view (c). Four years and 9 months post orbicularis myectomy via cervico-facial-temporal lifting at repose (d), smiling (e), and $45^{\circ}$ view (f). Note the persistence of the lower lines of the crows' feet at the lower aspect and also medial to the orbital rim where the myectomy does not extend into
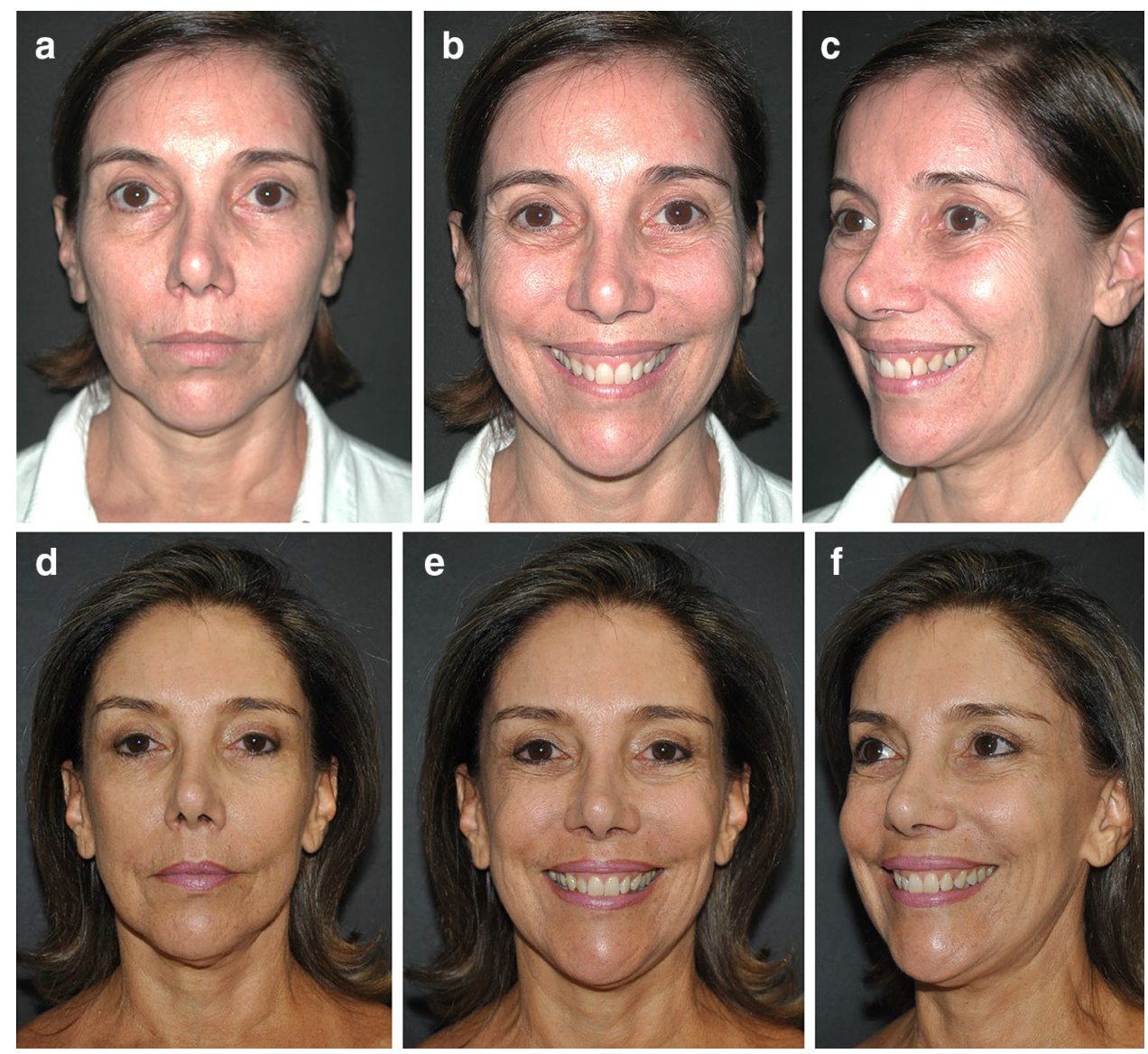

Regarding the technique that suspends and partially denervates the orbicularis oculi [16], we believe that the results can be unpredictable as it is difficult to get bilaterally symmetrical denervation. Furthermore, the consequent reinnervation is also unpredictable, and may result in asymmetry.

The visualization of the orbicularis oculi is dependent on the procedure performed. In myectomies performed via blepharoplasty and in those patients who have significant wrinkles extending to the inferior portion, less improvement was noted at the lower region. This can lead to lines remaining at the inferior portion (Fig. 11).

We believe that the myectomy provides definitive results if performed broadly to include the whole area where crows' feet wrinkles appear. Myectomy performed via lifting is much easier than via blepharoplasty and we recommend that colleagues who want to perform this technique start via the lifting approach.

The issue of postoperative contour deformity can be a problem with orbicularis myectomy. Some authors note that they "have not solved the problem of contour irregularity" [22] and other authors illustrate photos with residual lines but with high patient satisfaction [23]. This is similar to our series, where we have residual lines in patients who are satisfied. Using fat injection, we offer an approach to the correction of postoperative contour deformity that can be corrected at the initial surgery or at a later time.

Initially, we used to harvest small pieces of SMAS or en bloc rectangular-shaped fat grafts from the abdominal region. In three cases, we observed an overcorrection and we had to perform liposuction to decrease the graft volume. Subsequently, we did not use fat and noticed a hollowing in the area in three cases (Fig. 12). Two of these cases had delayed fat grafting. As we gained more experience, we have modified our procedure and now intraoperatively evaluate the need for fat grafting after muscle resection in every case. Typically, $3-5 \mathrm{ml}$ of injected fat is needed. This approach has the advantage of using liquid fat allowing sculpting and is less time consuming than en bloc fat grafting or SMAS grafting. We place micropore strips on the skin in an oblique and vertical direction at the grafted area to prevent fat migration and to aid even fat distribution especially in myectomies done via the blepharoplasty approach. In some cases, fat grafting was not required.

We believe that the orbicularis oculi myectomy is extremely important to achieve facial rejuvenation and we recommend this procedure to our colleagues. 

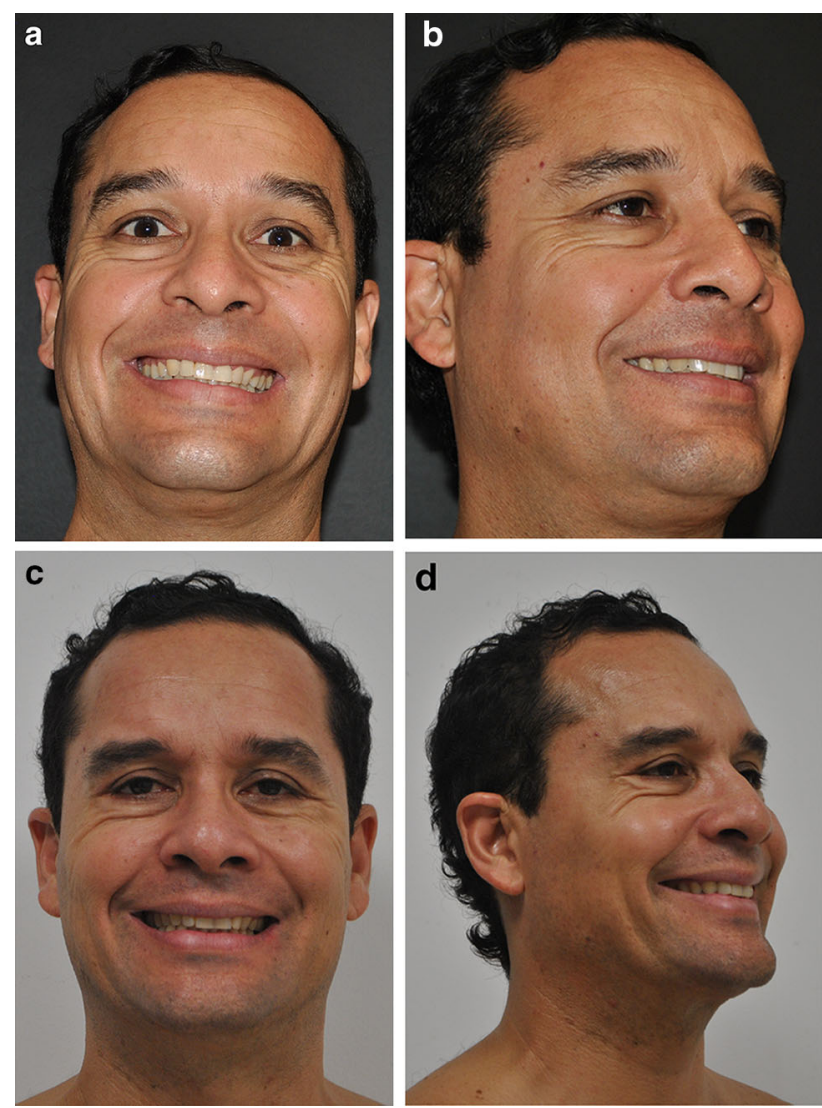

Fig. 11 Preoperative views of a 42-year-old male smiling (a) and $45^{\circ}$ view (b). 9 months post orbicularis myectomy via blepharoplasty approach: smiling (c) and $45^{\circ}$ view (d). Most of our male patients opt for the blepharoplasty approach thus making visualization of the orbicularis more difficult. The remnant inferior fibres of the orbicularis oculi result in the persistence of the lower lines of the crows' feet wrinkles
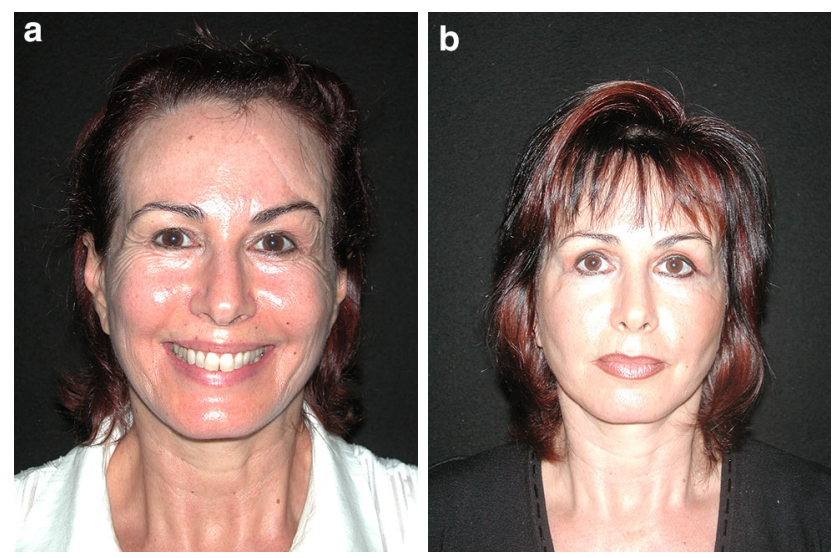

Fig. 12 Preoperative view showing crows' feet (a). One year postoperative view showing hollowing after myectomy (b). This was one of our earlier cases that was not fat grafted. This complication has largely been prevented by intraoperative fat injection

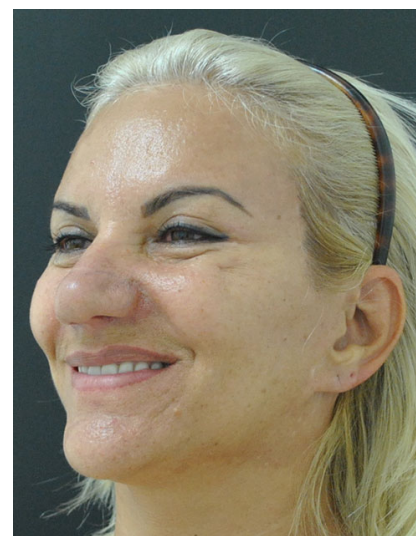

Fig. 13 Puckering seen postoperatively in the lower eyelid and most prominent when smiling. This could be a result of incomplete separation of skin from the muscle. This was managed conservatively by massaging

\section{Conclusion}

We highlight the evolution of our surgery for crows' feet wrinkle and discuss the complications and how we tried to prevent or manage them. We conclude that orbicularis oculi myectomy is an effective and long-term treatment for crows' feet wrinkles treatment, with a high satisfaction rate for patients.

Acknowledgments We thank Dr. Antonio Graziosi for the revision of the text. We also thank Eduardo Leite Fonseca and Larissa Pierri Carvalho for the evaluation of the pictures.

\section{Compliance with Ethical Standards}

Disclosure The first author receives royalties from Doctus Company that produces the Viterbo's dissectors.

\section{References}

1. Pitanguy I (2012) Facial rejuvenation surgery: a retrospective study of 8788 cases. Aesthet Surg J 32(4):393-412

2. Park DH, Han DG, Shim JS, Lee TJ, Park SU, Do ER, Choo KJ (2012) Analysis of the patterns of lateral canthal rhytids and reference for botulinum toxin treatment in orientals. Aesth Plast Surg 36:1211-1215

3. Lemperle G, Holmes RE, Cohen SR, Lemperle SM (2001) A classification of facial wrinkles. Plast Reconstr Surg 108:1735-1750

4. Tsukahara K, Osanai O, Kitahara T, Takema Y (2013) Seasonal and annual variation in the intensity of facial wrinkles. Skin Res Technol 19:279-287

5. Cula GO, Bargo PR, Nkengne A, Kollias N (2013) Assessing facial wrinkles: automatic detection and quantification. Skin Res Technol 19:e243-e251

6. Fujimura T, Hotta M (2013) A novel method to investigate the relationship between facial movements and wrinkle formation using particle image velocimetry. Skin Res Technol 19:e54-e59 
7. Carruthers J, Carruthers A (2009) Botulinum toxin in facial rejuvenation: an update. Dermatol Clin 27(4):417-425

8. Skoog TG (1974) Plastic surgery: new methods and refinements. Saunders, Philadelphia

9. Aston SJ (1980) Orbicularis oculi muscle flaps: a technique to reduce crows feet and lateral canthal skin fold. Plast Reconstr Surg 65:206-216

10. Connell B, Marten T (1993) Surgical correction of the crow's feet deformity. Clin Plast Surg 20:29

11. Camirand A (1993) Treatment of dynamic crow's feet while performing a blepharoplasty. Aesthet Plast Surg. 7:17

12. Bonatto A, Freitas AG, Mélega JM (2002) Myectomy of the orbicularis oculi muscle: a new procedure associated to blepharoplasty. Rev Soc Bras Cir Plast Sao Paulo. 17:27

13. Aston SJ (1981) Orbicularis oculi muscle flaps: a follow-up of the technique to reduce crow's feet and lateral canthal skin folds. Clin Plast Surg 8:679-686

14. Fogli AL (1995) Orbicularis muscleplasty and face lift: a better orbital contour. Plast Reconstr Surg 96:1560-1570 discussion 1571-1572

15. Cabbabe SW, Andrades P, Vasconez LO (2009) Lateral orbicularis oculi muscle plasty in conjunction with face lifting for periorbital rejuvenation. Plast Reconstr Surg 124(4):1285-1293
16. Guerrissi JO (2010) Periorbital rejuvenation: a safe subcutaneous approach to forehead, eyebrow, and orbicularis oculis muscle mobilization. Aesthet Plast Surg 34(2):147-152

17. Viterbo F (2003) New treatment for crow's feet wrinkles by vertical myectomy of the lateral orbicularis oculi. Plast Reconstr Surg 112:275

18. Viterbo F, Lutz BS (2006) Extended "C" myectomy of the lateral orbicularis oculi muscle-a safe and successful method for treatment of "crow's feet". Aesthet Surg J 26:131-135

19. Carvalho F, Silva V, Moreira A et al (2008) Definitive treatment for crow's feet wrinkles by total myectomy of the lateral orbicularis oculi. Aesthet Plast Surg 32:779

20. Ryu MH, Shin Y, Kahng D (2014) The surgical correction of crow's feet deformity with radiofrequency current. Aesthet Plast Surg 34:28

21. Park YJ, Jo YW, Bang SI et al (2007) Radiofrequency volumetric reduction for masseteric hypertrophy. Aesthet Plast Surg 31:42

22. Ryu MH, Kahng D, Shin Y (2014) Surgical correction of crow's feet deformity with radiofrequency current. Aesthet Surg J. 34(1):28-33

23. Connell BF, Marten TJ (1993) Surgical correction of the crow's feet deformity. Clin Plast Surg 20(2):295-302 